\title{
Mechanical Modeling and Analysis of Braided Wire Rope
}

\author{
ZHAO Xia, a WANG Hui ${ }^{1, b}$ ZHAO Ping ${ }^{1, c}$ KE ShengJun $^{1, d}$ SONG Bao ${ }^{1, e}$ \\ ${ }^{1}$ School of Mechanical Engineering, University of Jinan, Jinan 250022, China. \\ ajz1015zhaoxia@163.com, bme_wangh@ujn.edu.cn, ${ }^{\mathrm{c}} 18753136032 @ 163 . c o m$, \\ d18254130969@163.com, ${ }^{\mathrm{e}} 18366104129 @ 163 . c o m$
}

Keywords: Braided wire rope, mechanical modeling, stress, strain

Abstract. Braided wire rope has been widely used because of its high strength and high twist resistance, and its safety performance in service has been paid more and more attention.Based on the reasonable simplification of the wire rope, resorting to ellipse Four-Center-Arcs method, the deformation of the rope strands under tensile Load was analyzed. And on this basis, the mechanical model of braided wire rope under axial load was established. The stress, strain and the change curve of strand segments under different tensile loads were obtained by the numerical calculation with Matlab software. Calculation results show that: the axial stresses and strains of strand segments located on the small arc are less than that of the large arc. However, the corresponding shear stresses are greater than that of the large arc.

\section{Introduction}

There are two types of wire rope, which are lay wire rope and braided wire rope. The braided ropes consist of several round right and left lay strands which are symmetrically cross woven together. And the number of right lay strands and left lay strands is respectively half of the total. Because of its high strength and high twist resistance, braided wire rope is widely used in many kinds of conditions which require wire ropes have a high non-rotation.

With the extensive use of braided wire rope, it is repeatedly stretched, bent, twisted, subjected to load shock, overload, etc in the electric power construction project. So the phenomenons of fatigue failure and fracture of wire rope sometimes have occurred. Therefore its safety performance in service has been paid more and more attention.

At present, the scholars have made a deep research on the forming principle, mechanical modeling of lay wire rope and so on. ${ }^{[1-2]}$ By using differential geometry and elastic mechanics knowledge, the linear and nonlinear of lay wire rope structure were analyzed. And the finite element simulation of the wire rope under tension condition was carried out. ${ }^{[3-5]}$ However, the scholars have less research on the braided wire rope. Some scholars have made a preliminary study for braided wire rope from types, performance, structural characteristics and the problems existing in the processing and use, causes analysis of fracture, etc. But there are no discovery on mechanical modeling of braided wire rope. In order to explore the mechanical response of braided wire rope to the external loads, it is necessary to carry out mechanical modeling and investigate the stress and strain of braided wire rope under axial load. This will provide a theoretical basis for the failure mechanism analysis and maintenance of braided wire rope.

\section{Mechanical model of braided wire rope}

Basic geometric parameters. The geometric structure of the braided wire rope is shown in Fig.1. Due to the particularity of the structure, the stress state of the braided wire rope is very complicated.In order to facilitate the analysis, it is necessary to simplify the structure of the braided wire rope. The relative movement of the wires under the tensile load are very small because of the tight arrangement of wires in strand. Therefore, we ignore the influence of the wire and simplify the rope strand as a whole, as shown in Fig.2. 


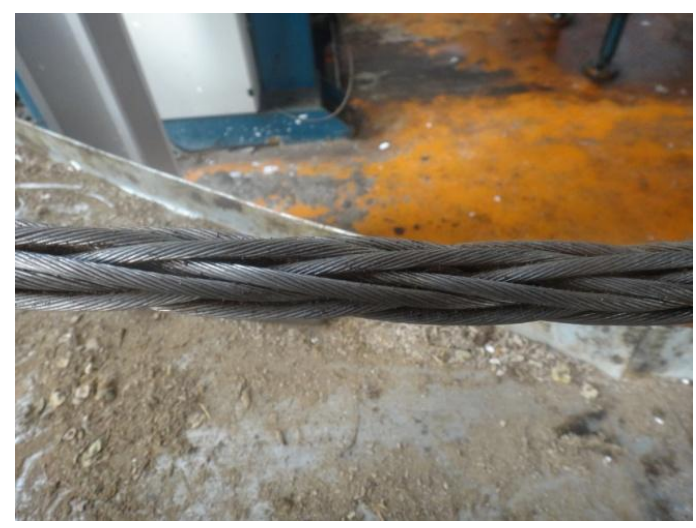

Fig.1 braided wire rope
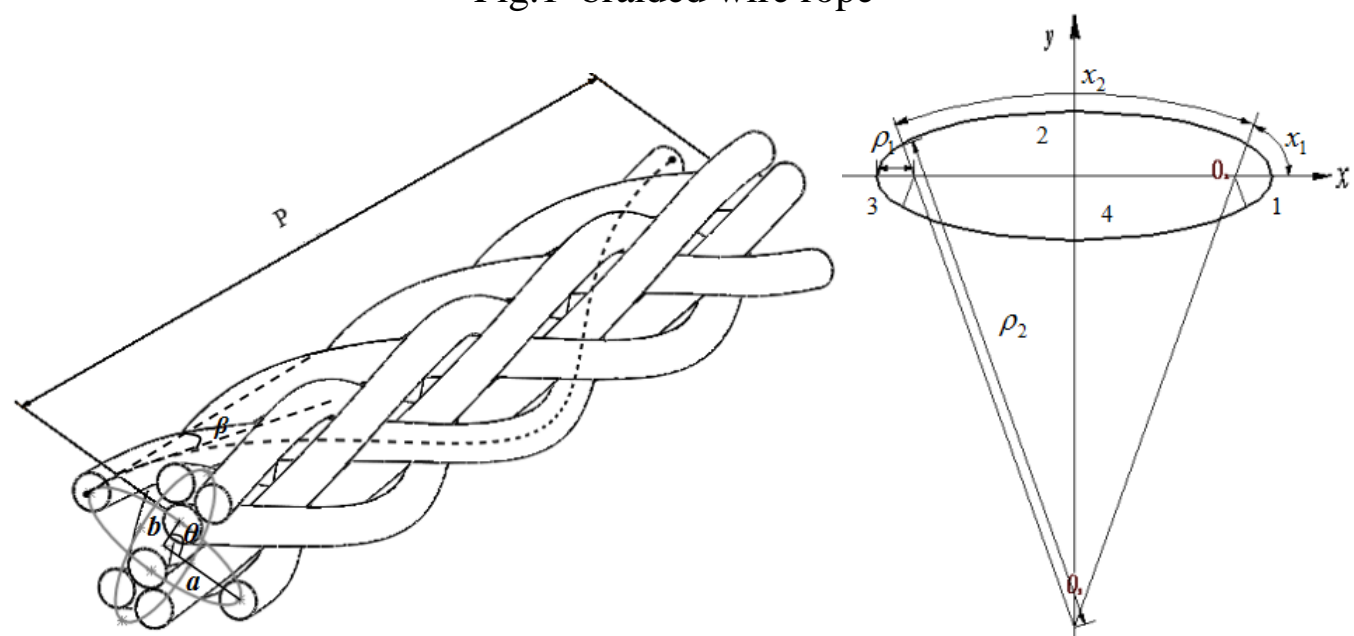

Fig.2 Simplified braided wire rope

Fig.3 Ellipse Four-Center Arcs

In the cross section, the projection of the center line of any rope strands in the braided wire rope is an ellipse. As shown in Fig.2, $a$ is the long half axis of the ellipse, $b$ is a short half axis of an ellipse, $\theta$ is the ellipse centrifugal angle, $P$ is the pitch of the braided wire rope(a point on the axis of strand rotates $360^{\circ}$ around the axis of the rope, the distance between the corresponding points), $\beta$ is the braided angle of rope strand( the angle between the center line of strand and the axial direction of the rope).In order to facilitate the analysis, the ellipse is divided into four sections according to the Four-Center-Arcs method, as shown in Fig.3. Here, $x_{1}$ is half of the small arc length, $x_{2}$ is a large arc length, $\rho_{1}$ is a small arc radius, $\rho_{2}$ is the radius of large arc segment.

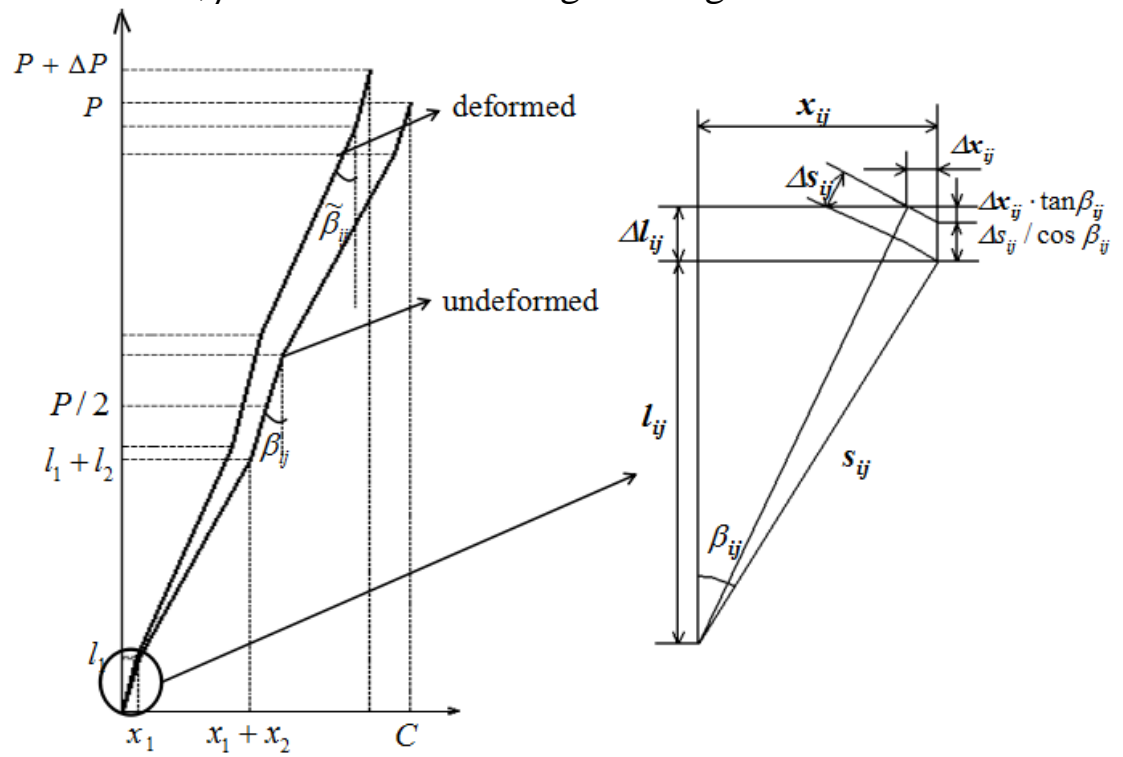

Fig.4 Strand deformation analysis 
The expansion line of each rope strand can be obtained by expanding the elliptic cylinder where the center lines of strands are located. It is well known that the wire rope will elongate under tensile load.Take the a rope strand as an example,its initial position is located at the ellipse's long axis,using the Four-Center-Arcs method, the approximate expansion lines of deformed and undeformed strands can be obtained.Select any section of the rope strand, and analyze its deformation as shown in Fig.4.

Here, $C$ is the circumference of an ellipse. $l_{1}, l_{2}$ respectively is the length of the rope corresponding to the arc length $x_{1}, x_{2} . \Delta P$ is the total elongation of wire rope in a pitch. $\beta_{i j}, \tilde{\beta}_{i j}$ respectively is the braided angle of the undeformed and deformed strand. $l_{i j}$ is the length of rope corresponding to the $j$ section of the rope strand $i$, Let $\Delta l_{i j}$ be its deformation after loading. $s_{i j}$ is length of rope strand,and $\Delta s_{i j}$ is its deformation after loading. $x_{i j}$ is arc length of an ellipse, $\Delta x_{i j}$ is its deformation after loading.

Mechanical modeling. By analyzing the approximate expansion lines of the rope strands in the unloaded and loaded state, the basic geometric parameters of the wire rope are obtained.And on the basis of this, analyzing the load condition of any section of strand,it can be carried out that:

$T_{i j}$ is the axial force of any section of strand along the rope. $T_{i j}$ can be expressed by the axial force along the strand $F_{i j}$ and the shear force of strand $Q_{i j}$ as follows

$$
T_{i j}=F_{i j} \cos \widetilde{\beta}_{i j}+Q_{i j} \sin \widetilde{\beta}_{i j} .
$$

Where the axial force along the strand is given by the following relation

$$
F_{i j}=\xi_{i j} \frac{E A \cos ^{2} \beta_{i j}}{\left(1+v \sin ^{2} \beta_{i j}\right)} .
$$

The shear force of strand can be written as

$$
Q_{i j}=\frac{\sin \tilde{\beta}_{i j}}{\tilde{\rho}_{i j}}\left\{\frac{E \pi R^{4}}{4}\left(\frac{\sin ^{2} \tilde{\beta}_{i j}}{\tilde{\rho}_{i j}}-\frac{\sin ^{2} \beta_{i j}}{\rho_{i j}}\right) \cos \tilde{\beta}_{i j}-\frac{E \pi R^{4}}{4(1+v)}\left(\frac{\sin \tilde{\beta}_{i j} \cos \tilde{\beta}_{i j}}{\tilde{\rho}_{i j}}-\frac{\sin \beta_{i j} \cos \beta_{i j}}{\rho_{i j}}\right) \sin \tilde{\beta}_{i j}\right\} .
$$

The braided angle of the deformed strand can be expressed as follows

$$
\sin \tilde{\beta}_{i j}=\frac{1+v \sin ^{2} \beta_{i j}-\xi_{i j} v \cos ^{2} \beta_{i j}}{1+v \sin ^{2} \beta_{i j}+\xi_{i j} \cos ^{2} \beta_{i j}} \sin \beta_{i j}, \quad \cos \tilde{\beta}_{i j}=\frac{\left(1+\xi_{i j}\right)\left(1+v \sin ^{2} \beta_{i j}\right)}{1+v \sin ^{2} \beta_{i j}+\xi_{i j} \cos ^{2} \beta_{i j}} \cos \beta_{i j} .
$$

The rotating radius of the deformed strand is derived as

$$
\tilde{\rho}_{i j}=\frac{2 x_{i j}}{\tilde{\theta}_{i j}}-\frac{2 \xi_{i j} v x_{i j} \cos ^{2} \beta_{i j}}{\left(1+v \sin ^{2} \beta_{i j}\right) \tilde{\theta}_{i j}} .
$$

Here, $\xi_{i j}$ represents the axial strain of any section of strand along the rope,$E$ represents rope strand elastic modulus, $A$ is strand cross section area, $v$ is Poisson's ratio, $\rho_{i j}, \tilde{\rho}_{i j}$ represents respectively the rotating radius of the undeformed and deformed strand segment. $\tilde{\theta}_{i j}$ is the rotating angle of deformed strand segment.

In addition,the axial stress of any section of strand along the strand $\sigma_{i j}$ is given by

$$
\sigma_{i j}=F_{i j} / A \text {. }
$$

The shear stress of strand $\tau_{i j}$ can be written as

$$
\tau_{i j}=Q_{i j} / A .
$$




\section{Numerical calculation and result analysis of the mechanical model}

Numerical calculation. According to the formulas of the 2.2 chapter and the basic parameter values of the braided wire rope, the axial tensile stress, shear stress and strain values of rope strand under different tensile loads can be programmed in Matlab, as shown in the Table 1.

Table 1 stress and strain values of strand under different axial loads

\begin{tabular}{cccllll}
\hline Axial loads of T(N) & 800 & 1000 & 1200 & 1600 & 2000 & 2400 \\
\hline$\xi_{1}$ & $1.08 \mathrm{e}-4$ & $1.34 \mathrm{e}-4$ & $1.6 \mathrm{e}-4$ & $2.12 \mathrm{e}-4$ & $2.64 \mathrm{e}-4$ & $3.16 \mathrm{e}-4$ \\
$\xi_{2}$ & $1.22 \mathrm{e}-4$ & $1.52 \mathrm{e}-4$ & $1.82 \mathrm{e}-4$ & $2.42 \mathrm{e}-4$ & $3.02 \mathrm{e}-4$ & $3.62 \mathrm{e}-4$ \\
$\sigma_{1}(\mathrm{Mpa})$ & 21.4306 & 26.5898 & 31.7490 & 42.0674 & 52.3858 & 62.7042 \\
$\sigma_{2}(\mathrm{Mpa})$ & 21.8086 & 27.1714 & 32.5342 & 43.2597 & 53.9853 & 64.7108 \\
$\tau_{1}(\mathrm{Mpa})$ & 4.1140 & 4.1291 & 4.1442 & 4.1744 & 4.2047 & 4.2349 \\
$\tau_{2}(\mathrm{Mpa})$ & 0.5879 & 0.5881 & 0.5882 & 0.5886 & 0.5889 & 0.5892 \\
\hline
\end{tabular}

The index 1 and 2 of the parameters in the table respectively refer to the section of strand corresponding the small arc and large arc.

Result analysis. According to the data of Table 1, the curve of the strain and the shear stress of the strand were obtained with the change of the external loads, as shown in Fig.5.

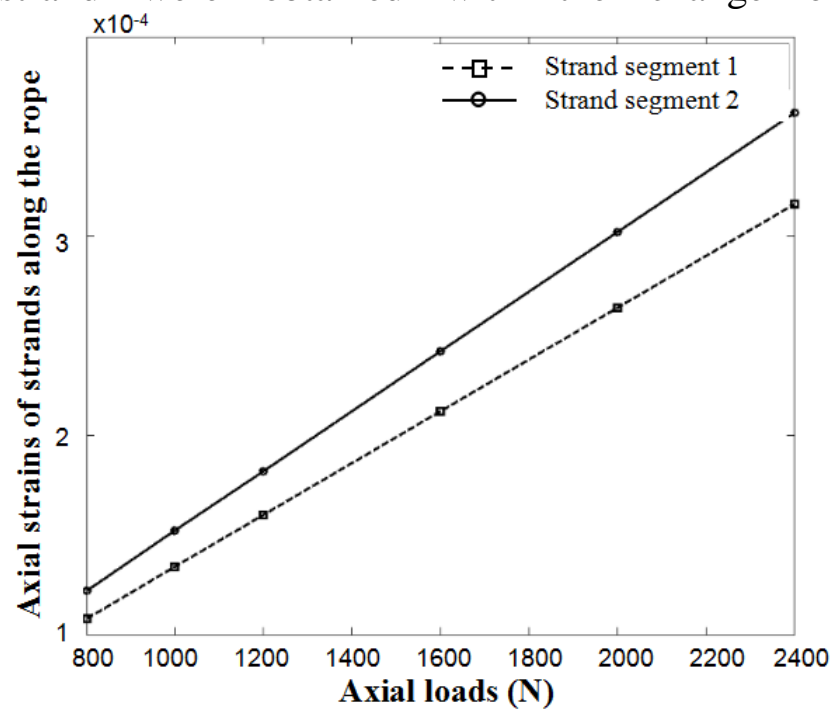

(a)

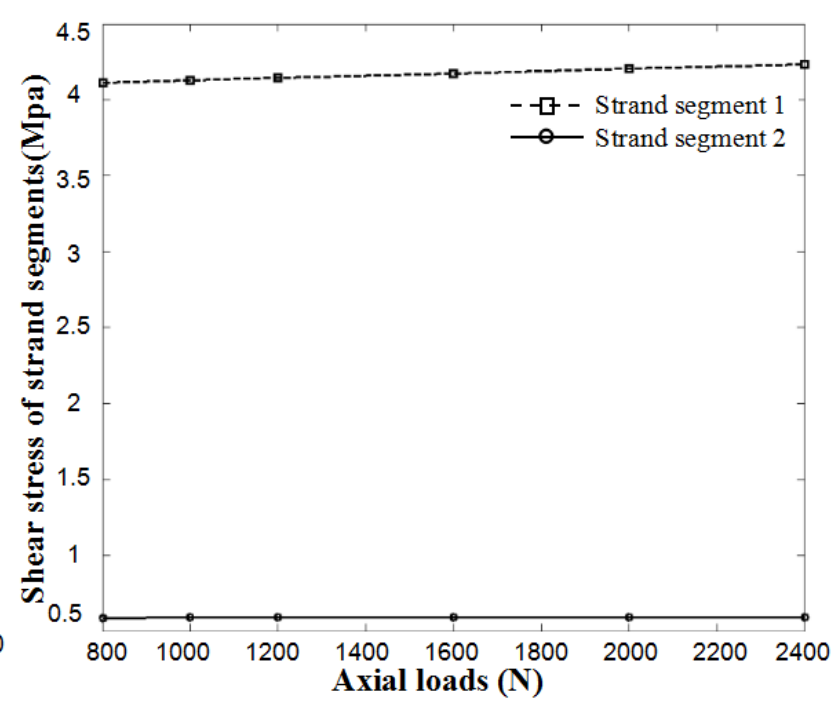

(b)

Fig.5 The strain and shear stress curves of strand segments with the change of external loads.(a)The strain curves of strand segments with the change of external loads.(b) The shear stress curves of strand segments with the change of external loads.

It can be seen from Table 1 and Fig. 5 that:

(1) Under the action of tensile load, in general ,the axial strain along the rope ,the axial stress along the strand and the shear stress increase linearly with the increase of loads.

(2) The axial strain along the rope and the axial stress along strand ,corresponding to the elliptic small arc, is smaller than that of the large arc.

(3) The shear stress of rope strand increases with the increase of external tensile loads, and the upward trend is not significant. Besides, the shear stress corresponding to the elliptic small arc is larger than that of the large arc. 


\section{Conclusions}

In this paper, the mechanical model of braided wire rope was calculated, and the results were analyzed, on the whole,the following conclusions were drawn:

(1) The strain and stress of the braided wire rope strand increase linearly with the increase of tensile loads.But the upward trend of the shear stress of rope strand is not significant.

(2) The axial strain and stress of strand segment corresponding to the elliptic small arc, is smaller than that of the large arc.But the shear stress of strand segment corresponding to the elliptic small arc is larger than that of the large arc.

(3) The stress and strain of rope strand under axial loads were calculated and analyzed in this paper, which provides a theoretical basis for the failure mechanism analysis and safe use of the braided wire rope.

\section{Acknowledgements}

The work was financially supported by the Science and Technology Development Project of Shandong Province(2014GGX103016).

\section{References}

[1] K Feyrer.Wire Ropes-Tension,Endurance, Reliability[M].Berlin:Springer,2007:34-204.

[2] Leech CM. The Modelling and Analysis of the Mechanics of Ropes[M]. Berlin Springer2013; $5-113$.

[3] Ivan Argatov. Response of a wire rope strand to axial and torsional loads: Asymptotic modeling of the effect of interwire contact deformations[J]. International Journal of Solids and Structures. 2011, 48: 1413 -1423.

[4] Yujie Yu, Zhihua Chen and Hongbo Liu, etal. Finite element study of behavior and interface force conditions of seven-wire strand under axial and lateral loading $[\mathrm{J}]$. Construction and Building Materials 2014,66: 10-18.

[5] Vladimir Ivanco, Stanislav Kmet, Gabriel Fedorko. Finite element simulation of creep of spiral strands[J]. Engineering Structures2016,117: 220-238. 